A N N A L E S Annales de Bretagne et des Pays de l'Ouest

Anjou. Maine. Poitou-Charente. Touraine

118-1| 2011

Varia

\title{
Le patrimoine religieux de la manufacture des toiles «bretagnes »
}

Prospérité toilière et ambition fabricienne en Centre-Bretagne

(1650-1790)

\section{Anthony Guillemot}

\section{(2) OpenEdition}

\section{Journals}

Édition électronique

URL : http://journals.openedition.org/abpo/1896

DOI : 10.4000/abpo.1896

ISSN : 2108-6443

\section{Éditeur}

Presses universitaires de Rennes

\section{Édition imprimée}

Date de publication : 10 avril 2011

Pagination : 87-111

ISBN : 978-2-75351410-2

ISSN : 0399-0826

Référence électronique

Anthony Guillemot, "Le patrimoine religieux de la manufacture des toiles « bretagnes » », Annales de Bretagne et des Pays de l'Ouest [En ligne], 118-1 | 2011, mis en ligne le 10 avril 2013, consulté le 01 mai 2019. URL : http://journals.openedition.org/abpo/1896 ; DOI : 10.4000/abpo.1896 


\title{
Le patrimoine religieux de la manufacture des toiles «bretagnes »: prospérité toilière et ambition fabricienne en Centre-Bretagne (1650-1790)
}

\author{
Anthony GUILLEMOT \\ Étudiant en master - I'université Rennes 2 Haute-Bretagne \\ enseignant du secondaire
}

En Bretagne, la constitution d'un patrimoine religieux monumental ${ }^{1}$ grâce aux profits du commerce toilier est bien connue et remarquable-


semble exceptionnel par la splendeur et l'ostentation des grands enclos paroissiaux, l'on peut se demander s'il est un cas isolé dans une province qui compte d'autres secteurs et d'autres moments de prospérité toilière. $\mathrm{Au} x \mathrm{XIII}{ }^{\mathrm{e}}$ siècle en particulier, dans un contexte breton par ailleurs difficile, il est une activité qui prospère en Centre-Bretagne : celle de la production des toiles de lin dites "bretagnes», production de luxe, réputée pour sa qualité. Le dynamisme des toiles «bretagnes », fortement attesté à partir du

1. Je remercie bien sincèrement Georges Provost de l'aide qu'il m'a apportée pour la rédaction et la relecture de cet article. Celui-ci reprend les principales conclusions du mémoire de Master 2 que j'ai soutenu à l'université de Rennes 2 en juillet 2007 sous sa direction : GUILLEMOT, Anthony, Prospérité toilière et chantiers paroissiaux dans les paroisses rurales de la manufacture des toiles « bretagnes » 1650-1830, 357 p. Ce mémoire fournit, en annexe II (p. 316-336), I'inventaire des chantiers paroissiaux recensés dans le territoire de la manufacture.

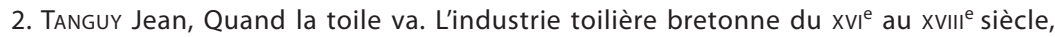
Rennes, Apogée, 1994, 158 p. Pour ce qui concerne le Léon toilier, voir aussi : ELÉGOËT, Louis, Les Juloded. Grandeur et décadence d'une caste paysanne en Basse-Bretagne, PUR, 1996, 292 p.; TANGUY, Stéphane, Les enclos et I'argent : I'arrière-plan financier des réalisa-

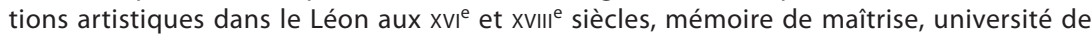
Brest, 1985, 135 p.; PORHEL, Jean-Luc, Les chantiers paroissiaux dans le Léon du $x v^{\mathrm{e}}$ au $\mathrm{XVIII}$ e siècles, mémoire de maîtrise sous la direction de Jean Tanguy, université de Brest, 1982, 157 p.; CHAURIS, Anne, Le poids financier des enclos léonards, mémoire de maîtrise sous la direction de Fañch Roudaut, université de Brest, 1996, 399 p. Voir aussi MARTIN, Jean et Pellerin, Yvon (dir.), Du lin à la toile. La proto-industrie textile en Bretagne, Rennes, PUR, 2008, 336 p. 
dernier quart du $\mathrm{XvI}^{\mathrm{e}}$ siècle ${ }^{3}$, s'explique par leur écoulement massif sur les marchés des colonies espagnoles d'Amérique, via l'intermédiaire de SaintMalo puis de Cadix. Bien connu à partir du milieu du XvIII ${ }^{\mathrm{e}}$ siècle, le volume des exportations va croissant jusque vers 1775 et permet de dégager de substantiels profits, comme l'a démontré Jean Martin ${ }^{4}$. Cette manufacture faisait travailler 35000 personnes à la veille de la Révolution, dans une vaste zone rassemblant une cinquantaine de paroisses entre Pontivy, SaintBrieuc, Corlay et Moncontour.

Comme dans les autres régions toilières ayant connu un temps de prospérité, la question se pose de savoir si, dans cette manufacture des "bretagnes", la richesse a laissé des traces et un patrimoine monumental. En Léon, la réponse s'impose du fait de l'existence des somptueux enclos paroissiaux mais ce lien entre prospérité et chantiers paroissiaux n'est pas systématique dans d'autres zones toilières. Dans la région de Vitré, qui connut une activité toilière importante entre le $x V I^{e}$ et le $x I x^{e}$ siècle, grâce notamment aux "noyalles », toiles de chanvre destinées essentiellement à fournir les voiles des navires, les traces de la prospérité sont ainsi plus difficiles à retrouver. S'agissant du patrimoine religieux, Yann Lagadec et Delphine Pointeau ${ }^{5}$ observent que les transformations de l'église NotreDame de Vitré reflètent, en milieu urbain, la prospérité négociante issue des toiles, mais qu'il faut se montrer plus prudent concernant son impact sur les campagnes. Au $\mathrm{XVII}^{\mathrm{e}}$ siècle, les retables lavallois ${ }^{6}$ se sont certes multipliés dans les paroisses toilières mais il en a été souvent de même dans celles qui ne l'étaient pas. L'effet de mode suffit probablement à rendre compte de leur grande diffusion. En outre, Yann Lagadec et Delphine Pointeau notent que les revenus de ces paroisses restaient modestes et surtout que la part des offrandes faites par les habitants y était bien maigre ${ }^{7}$, alors que c'était justement l'accroissement spectaculaire de celles-ci qui avait permis aux paroisses du Léon de se lancer dans des dépenses somptuaires ${ }^{8}$.

3. La première attestation significative est fournie en 1686 par le mémoire de l'intendant Patoulet sur le commerce de Cadix (LESPAGnol, André, Messieurs de Saint-Malo. Une élite négociante au temps de Louis XIV, Saint-Malo, L'Ancre de Marine, 1990, p. 428-429).

4. Martin, Jean, Toiles de Bretagne. La manufacture de Quintin, Uzel et Loudéac, 16701830, Rennes, PUR, 1998, 374 p.; du même, "La manufacture des toiles bretagnes du XVII au XIX siècle », dans Martin Jean et PelLeRIN Yvon (dir.), Du lin à la toile..., op. cit., p. 231-271.

5. Lagadec, Yann et Pointeau Delphine, « La proto-industrie dans les campagnes des environs de Vitré ( $\mathrm{xVI} \mathrm{e}^{\mathrm{e}}-\mathrm{XI} \mathrm{x}^{\mathrm{e}}$ siècles) : un modèle spécifique de production? ", Mémoires de la Société d'histoire et d'archéologie de Bretagne, tome LXXXIV, 2006, p. 181-207.

6. Sur ce type de retable voir : MÉNARD, Michèle, Une Histoire des mentalités religieuses aux XVII ${ }^{\mathrm{e}}$ et $\mathrm{XVII \textrm {I } ^ { \mathrm { e } }}$ siècles. Mille retables de l'ancien diocèse du Mans, Paris, Beauchesne, 1980, 467 p.; SALBERT Jacques, Les Ateliers des retabliers lavallois aux XVII et XVIII ${ }^{\mathrm{e}}$ siècles. Étude historique et artistique, Paris, Klincksieck, 1976, 540 p. Sur la question des retables, voir aussi : RESTIF, Bruno, La Révolution des paroisses. Culture paroissiale et Réforme catholique en Haute-Bretagne aux XVI $l^{\mathrm{e}}$ et XVII ${ }^{\mathrm{e}}$ siècles, Rennes, PUR, 2006, $418 \mathrm{p}$.

7. Yann Lagadec et Delphine Pointeau évoquent l'exemple de Louvigné-de-Bais où 67 à $75 \%$ des revenus de la fabrique entre le $x V l^{e}$ et le $x V I I{ }^{e}$ siècle venaient de la dîme : c'était cela, bien plus que la toile, qui a permis les grandes dépenses faites pour l'église.

8. TANGUY, Jean, Quand la toile va..., op. cit., p. 114-117. 
Au vu de ces diversités, la question se pose donc de l'existence ou non d'un lien entre prospérité toilière et chantiers paroissiaux en CentreBretagne. La trace patrimoniale la plus remarquable laissée par les toiles "bretagnes » est celle des hôtels particuliers bâtis par les marchands de toiles, en particulier à Quintin ${ }^{9}$. Mais qu'en est-il ici des chantiers paroissiaux, beaucoup moins apparents dans le paysage comme dans I'historiographie ${ }^{10}$ ? Sans doute faut-il rappeler que la structure propre de la manufacture des « bretagnes » est différente de celle des « crées » du Léon. L'essentiel des profits issus du commerce est ici capté par les négociants de Saint-Malo ainsi que par une élite de grands marchands de toiles qui dominent toute l'activité à l'intérieur de la manufacture ${ }^{11}$. Les tisserands eux-mêmes n'ont souvent pas d'activité agricole de complément et sont étroitement soumis à l'élite marchande. Les ordonnances royales de 1736 et 1779 qui réglementent la fabrication et la commercialisation des toiles «bretagnes» renforcent encore leur soumission ${ }^{12}$.

Dans ces conditions, la prospérité collective est ici moins remarquable car davantage concentrée entre les mains d'une élite restreinte. Peut-on dès lors considérer que les paroisses en ont été tenues à l'écart? Bon nombre des anciennes paroisses toilières possèdent encore des édifices religieux datant de la prospérité ${ }^{13}$, même si ceux-ci n'ont pas la notoriété des enclos léonards. Est-il possible, transposant la démarche mise en œuvre en Léon, de retrouver dans les courbes d'offrandes faites aux paroisses du CentreBretagne le reflet de la prospérité toilière? Peut-on établir un rapport entre des chantiers paroissiaux et le mouvement de la prospérité, dans le temps comme dans l'espace? On sait en effet que l'activité de la manufacture fut marquée par des variations chronologiques, selon le développement des différents marchés qui la composaient : ainsi les paroisses et trèves ${ }^{14} \mathrm{du}$ marché de Quintin se sont-elles enrichies les premières, avant que la prospérité ne s'étende peu à peu vers le marché de Loudéac qui domine l'activité dans la seconde moitié du $\mathrm{xvIII}^{\mathrm{e}}$ siècle ${ }^{15}$. Notre objectif serait ainsi de démontrer que si les traces de cette prospérité sur le patrimoine religieux sont ici moins flamboyantes qu'en Léon, elles n'en sont pas moins réelles.

Des facteurs favorables, liés à la toile, ont en effet offert à certaines paroisses de réelles potentialités d'investissement dans les chantiers religieux : la situation géographique par rapport aux marchés mais aussi la

9. MARTIN, Jean, Toiles de Bretagne..., op. cit., p. 252-271.

10. Guère plus de deux pages dans ibidem, p. 251-253.

11. MARTIN, Jean, (Toiles de Bretagne..., op. cit., p. 174) estime cette élite de grands marchands à environ 80 individus dans la seconde moitié du XVIII ${ }^{\mathrm{e}}$ siècle.

12. Ibidem, p. 88.

13. Couffon, René, «Répertoire des églises et chapelles du diocèse de Saint-Brieuc et de Tréguier », Mémoires de la Société d'Émulation des Côtes-du-Nord, 1939-1941.

14. Une trève désigne une succursale d'une paroisse. Le desservant ecclésiastique de la trève, le curé, est nommé par le recteur de la paroisse mère qui tient aussi les registres paroissiaux. La trève dispose de sa propre église et de ses fonts baptismaux.

15. MARTIN, Jean, Toiles de Bretagne..., op. cit., p. 118. 
présence d'un milieu marchand entreprenant et fortuné. II importera donc ici de joindre à l'approche économique une approche plus sociale centrée sur les marchands de toiles ${ }^{16}$. Pour identifier les chantiers et le rôle que la toile et les marchands ont pu y tenir, il est nécessaire de croiser divers types de sources : comptes de fabriques, rôles d'imposition et registres de délibérations, sans oublier les monuments eux-mêmes quand ils nous sont parvenus. Lorsque, en dépit des lacunes, une telle conjonction documentaire est possible, elle permet de mettre en évidence un lien entre la toile et les chantiers paroissiaux en Centre-Bretagne. Toutefois, on verra que la nature de ce lien doit être envisagée avec nuances : apparemment direct ici, plus indirect là, il frappe surtout par son caractère non systématique et il ne saurait, à lui seul, rendre compte de toute la réalité.

\section{Des conjonctures fabriciennes contrastées}

Dans le « modèle » léonard, le premier - car le plus immédiat - signe d'enrichissement d'une paroisse passe par l'augmentation des offrandes que les paroissiens accordent à leur église et par l'accroissement du trésor de la fabrique. Le niveau atteint par cet enrichissement et la manière dont il évolue dans le temps forment ce que Jean Tanguy a appelé la « conjoncture fabricienne ${ }^{17} »$. Les sources paroissiales, particulièrement les comptes de fabrique, sont de première importance pour la percevoir, si tant est que les fonds conservés sont assez continus pour permettre de suivre la situation sur le long terme.

Dans la « charge » de la fabrique - c'est-à-dire ses revenus - il est possible de distinguer les offrandes faites en argent (qui incluent la " tasse courante " passée pendant le service religieux) et celles faites en nature. Etudiant le Léon, Jean Tanguy a noté que parmi ces dons en nature, la place du fil établissait de manière directe le lien entre l'enrichissement de la paroisse et la prospérité toilière ${ }^{18}$. Plus globalement, l'enrichissement des paroissiens par le commerce des toiles leur permettait d'être ensuite plus généreux envers les fabriques.

Les comptes dont nous disposons pour les paroisses ou trèves de la manufacture des «bretagnes » ne sont, hélas, pas aussi éloquents. La simplification de leur rédaction au $\mathrm{XVIII}^{\mathrm{e}}$ siècle, dans le droit fil d'une évolution entamée dès le siècle précédent ${ }^{19}$, ne permet plus de connaître précisément la composition de la « charge » des fabriques. Rares sont les comptes qui mentionnent le détail des dons en nature et leurs valeurs respectives. Quand c'est le cas, les principaux dons en nature sont en général le blé ou

16. Sur l'approche sociale des zones toilières, voir également les travaux de : LAGADEC, Yann, Pouvoir et religion au village : la vie paroissiale à Loudéac, Cadélac, Trévé et SaintCaradec au XVIII ${ }^{\mathrm{e}}$ siècle (vers 1680-1790), mémoire de maîtrise, université de Rennes 2, sous la direction d'Alain Croix, 1991, 409 p.; ELÉGOËT, Louis, Les Juloded..., op. cit.

17. TANGUY, Jean, Quand la toile va..., op. cit., p. 91-95, 110-114.

18. Ibidem, p. 95.

19. RESTIF, Bruno, La Révolution des paroisses..., op. cit. 
le beurre. Le fil est rarement identifié seul : il est le plus souvent inclus dans une catégorie rassemblant les "hardes et fils", catégorie dont le revenu très faible suggère bien que la part du fil se réduit à peu de chose ${ }^{20}$.

Plus que dans la donation directe de fil, la prospérité doit donc être recherchée dans l'augmentation d'ensemble des offrandes faites aux fabriques. L'appréciation du montant global des offrandes doit, naturellement, tenir compte de différents éléments de pondération : la chronologie et notamment les effets de l'inflation du XVIII siècle, la présence éventuelle de confréries et de chapelles susceptibles d'absorber une partie de la générosité des paroissiens, le volume démographique de chaque communauté ${ }^{21}$. Ces préalables posés, il apparaît qu'une croissance significative des offrandes peut s'observer en plusieurs paroisses de la manufacture mais avec bien des inégalités et, parfois, des interrogations quant au rapport direct avec la toile.

Tableau 1 - Évolution des niveaux moyens des offrandes annuelles en livres dans les paroisses de la manufacture des toiles « bretagnes » (Les périodes indiquées servent de cadre approximatif : elles sont adaptées selon les cas et les lacunes des sources. Les chiffres entre parenthèses correspondent au nombre de comptes utilisés pour constituer les moyennes.)

\begin{tabular}{|c|c|c|c|c|c|c|}
\hline \multirow[t]{2}{*}{ Paroisses } & \multicolumn{2}{|c|}{$\begin{array}{c}\text { Niveau des offrandes } \\
\text { vers } 1670-1680\end{array}$} & \multicolumn{2}{|c|}{$\begin{array}{c}\text { Niveau des offrandes } \\
\text { vers } 1740-1750\end{array}$} & \multicolumn{2}{|c|}{$\begin{array}{c}\text { Niveau des offrandes } \\
\text { vers } 1780-1790\end{array}$} \\
\hline & $\begin{array}{l}\text { valeur en } \\
\text { livres }\end{array}$ & $\begin{array}{c}\text { nombre de } \\
\text { cas }\end{array}$ & $\begin{array}{c}\text { valeur en } \\
\text { livres }\end{array}$ & $\begin{array}{l}\text { nombre de } \\
\text { cas }\end{array}$ & $\begin{array}{l}\text { valeur } \\
\text { en livres }\end{array}$ & $\begin{array}{l}\text { nombre } \\
\text { de cas }\end{array}$ \\
\hline Le Bodéo & 73 & (8) & 60 & (5) & 62 & (5) \\
\hline Boqueho & 94 & (9) & 11 & (8) & 25 & (6) \\
\hline Cadélac & & & 208 & (7) & 230 & (8) \\
\hline Caurel & & & 85 & (3) & 203 & (6) \\
\hline Cohiniac & & & 16 & (11) & 12 & (8) \\
\hline Le Foeil & 34 & (3) & 44 & (10) & 57 & (6) \\
\hline Le Leslay & & & 235 & (4) & 251 & (9) \\
\hline Plaine-Haute & 102 & (5) & 8 & (11) & 7 & (5) \\
\hline Plœuc & 160 & (5) & 241 & (10) & 280 & (11) \\
\hline Le Quillio & 325 & (10) & & & & \\
\hline Saint-Brandan & & & 83 & (5) & 80 & (3) \\
\hline Vieux-Bourg & & & 904 & (10) & 716 & (11) \\
\hline
\end{tabular}

Ce tableau global montre qu'il n'y a pas de prospérité homogène, les niveaux des paroisses étant très différents. S'il y a prospérité, elle ne soutient pas la comparaison avec celle du Léon (rappelons les 2000 livres d'offrandes annuelles perçues par la paroisse de Saint-Thégonnec vers 1670), excepté peut-être pour le Vieux-Bourg. Quant au lien avec la prospérité toi-

20. Ainsi, au Leslay en 1757, sur les 247 livres rapportées par toutes les offrandes cette année-là, la part du fil est de 9 livres (Arch. dép. des Côtes-d'Armor, 20 G 214).

21. Les écarts de dons importants entre des paroisses peuvent tenir davantage à une différence de population qu'aux inégalités de la prospérité toilière. 
lière, il requiert une approche plus précise qui peut être menée à l'échelle des paroisses les mieux documentées.

L'exemple le plus intéressant est ici fourni par les comptes de fabrique de la paroisse du Bodéo qui présentent jusqu'en 1669 des listes précises de donateurs avec la somme correspondante ${ }^{22}$. L'augmentation de leur nombre entre 1645 et 1669 participe à l'augmentation des revenus de la fabrique ces années-là et coïncide avec le début de la prospérité toilière dans cette partie nord de la manufacture ${ }^{23}$. Ainsi, la moyenne du nombre de donateurs passe-t-elle de 65 entre 1645 et 1657 à 89 entre 1658 et 1669 .

Malgré cette conjoncture favorable, le niveau des offrandes du Bodéo reste modeste : il ne dépasse les 100 livres qu'en 1664 et en 1700 . Dans le premier quart du $x \mathrm{VIII}{ }^{\mathrm{e}}$ siècle, les offrandes représentent en général le tiers des revenus de la paroisse, avec une moyenne de 55 livres entre 1700 et 1728 , dans un contexte favorable au commerce des "bretagnes », marqué notamment par l'aventure du trafic interlope lors de la guerre de succession d'Espagne ${ }^{24}$. Après 1750 , le niveau des dons stagne, voire régresse si I'on tient compte de l'inflation. Par ailleurs, à partir de 1710, une partie de la générosité des paroissiens se trouve détournée par la création d'une confrérie du Rosaire ${ }^{25}$. II faudrait donc ajouter les dons faits à la confrérie à ceux de l'église pour appréhender dans son ensemble l'ampleur de la générosité des paroissiens, mais les archives conservées ne le permettent pas. Toutefois, il apparaît que si la puissance fabricienne reste modeste, la conjoncture que dessine le mouvement des offrandes correspond de manière très directe au développement du commerce toilier et à ses aléas ${ }^{26}$.

La trève du Quillio, dans le marché d'Uzel, connaît, elle aussi, une augmentation de ses offrandes dès la seconde moitié du $\mathrm{xvII}^{\mathrm{e}}$ siècle. La moyenne passe ainsi de 200 livres entre 1654 et 1668 à plus de 300 livres entre 1669 et $1682^{27}$. Hélas, les comptes ne se poursuivent pas au-delà, ce qui empêche de suivre l'évolution des offrandes dans cette trève qui connaît au XVIII siècle l'apogée de son activité toilière.

Ces conjonctures fabriciennes résolument orientées à la hausse n'ont cependant aucun caractère général car il est dans la manufacture des « bretagnes " d'autres paroisses qui ne présentent pas, quoique de taille parfois imposante, un même niveau et une même croissance. Ainsi à Plœuc,

22. Arch. dép. des Côtes-d'Armor, 20 G 10.

23. Le Bodéo appartient au marché de Quintin qui est le premier à s'être développé. Cf. MARTIN, Jean, Toiles de Bretagne... op. cit.

24. Ibidem, p. 71.

25. Les comptes de cette confrérie sont conservés (Arch. dép. des Côtes-d'Armor, 20 G 11 ), mais de façon trop lacunaire pour permettre d'établir une courbe des dons. Elle reçoit 95 livres en 1718 mais il y a peu de chance pour qu'un tel niveau - reflet de l'enthousiasme qui accompagne sa création - se soit maintenu par la suite.

26. Aléas d'autant plus nombreux que la toile est un produit d'exportation donc soumis à l'instabilité du contexte international.

27. Arch. dép. des Côtes-d'Armor, 20 G 703/704. 
Graphique 1 - Valeur moyenne en livres des offrandes faites à la fabrique du Bodéo

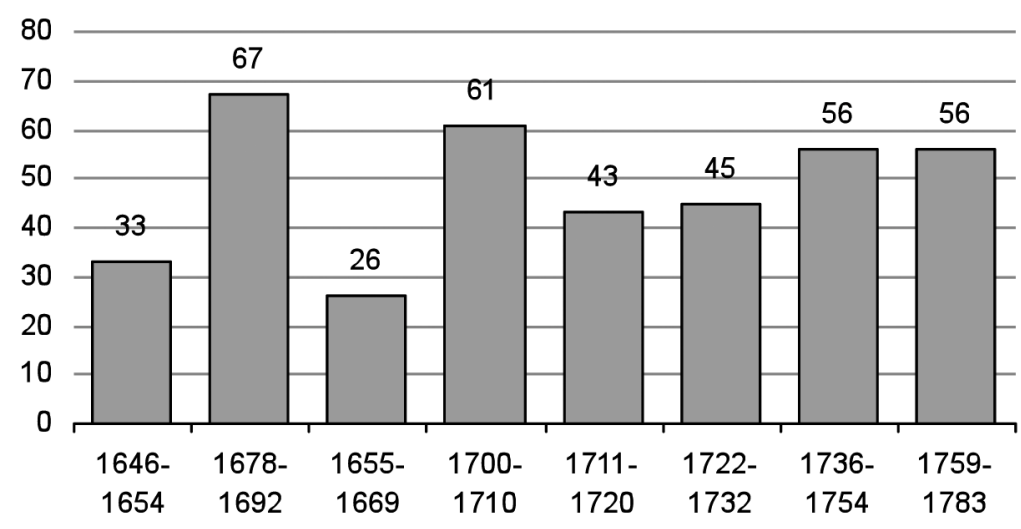

Graphique 2 - Valeur moyenne en livres des offrandes faites à la fabrique du Quillio

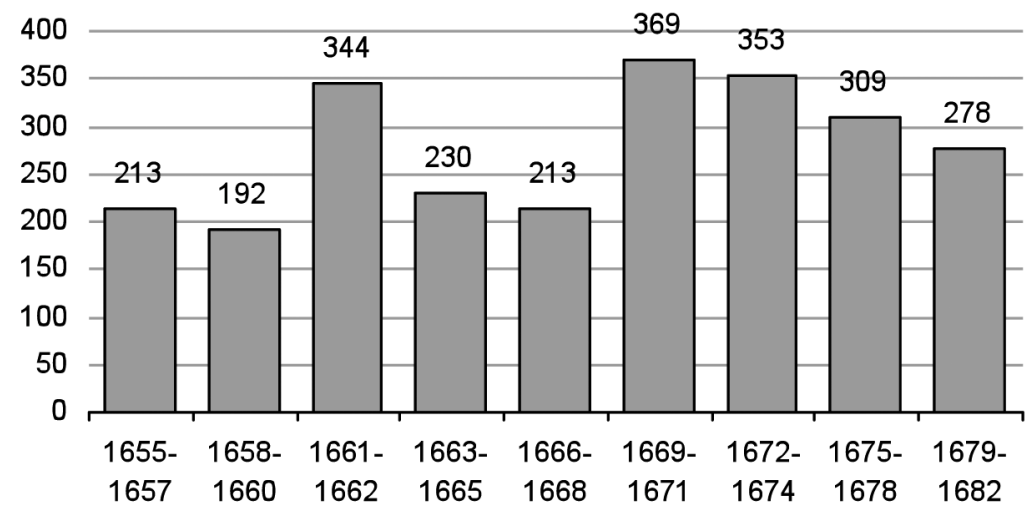

grosse paroisse qui comptait 5464 habitants en $1790^{28}$. Au XVIII siècle, le niveau d'offrandes à la fabrique était à peine équivalent à celui du Quillio un siècle plus tôt. De même, la trève du Fœil, malgré ses 2400 habitants en $1790^{29}$, n'a qu'une moyenne de 55 livres $^{30}$ d'offrandes annuelles entre 1768 et 1788 , soit le niveau du Bodéo au début du siècle avec une population bien moindre. Dans ces deux cas, la faiblesse des dons peut s'expliquer

28. MARTIN, Jean, Toiles de Bretagne..., op. cit., p. 232.

29. Ibidem.

30. Arch. dép. des Côtes-d'Armor, 20 G 77. 
par le niveau élevé des rentes (ou d'autres rentrées comme les luminaires) perçues par la fabrique et qui fait sa richesse relative. Cela ne signifie pas que les habitants ne profitent pas du commerce toilier et ne s'enrichissent pas car il y a des marchands de toiles à Plœuc ${ }^{31}$. Simplement, la fabrique dispose déjà d'importants revenus, ce qui n'incite pas les paroissiens à se montrer particulièrement généreux.

Au nord de la manufacture, dans le marché de Quintin, la faiblesse des offrandes est également la situation la plus fréquente au XVIII ${ }^{\mathrm{e}}$ siècle. Tel est le cas de Plaine-Haute, de Saint-Donan ou encore de Cohiniac. Ce dernier exemple est particulièrement révélateur. Les moyennes d'offrandes ne culminent qu'à 22 livres entre 1700 et $1710^{32}$. Le niveau décroît ensuite à ce point qu'après 1778 les comptes les incluent sans distinction dans les luminaires. L'essentiel des revenus est constitué par les rentes ${ }^{33}$ mais cette paroisse comptait aussi deux chapelles et deux confréries qui reçoivent plus de dons que l'église, les meilleures moyennes s'établissant autour de 50 livres. Ce sont là des paroisses à la fortune fabricienne très limitée, où rien ne semble marquer l'impact de la prospérité toilière comme au Quillio ou au Bodéo. Précisons que l'on se trouve à la frontière nord de la manufacture, dans le marché de Quintin où les marchands de toiles sont presque tous rassemblés dans la cité où ils concentrent les profits du commerce toi$\operatorname{lier}^{34}$. Les paroisses les plus septentrionales n'ont donc certainement reçu qu'un faible écho de cette prospérité toilière qui ailleurs peut permettre des enrichissements notables.

À ce titre, la paroisse du Vieux-Bourg, paroisse rurale voisine de Quintin, constitue un cas paradoxal, d'interprétation délicate. Les comptes conservés pour la période $1716-1788^{35}$ montrent une importante augmentation, surtout dans la seconde moitié du siècle, des offrandes faites à la fabrique de cette paroisse qui comptait 1370 habitants en $1790^{36}$. Elles vont même atteindre des niveaux inédits pour une paroisse rurale de la manufacture des «bretagnes » puisque entre 1750 et 1775 , le niveau des offrandes dépasse à six reprises les 1000 livres. On ne trouve de tels niveaux que dans les paroisses urbaines de la manufacture comme à Saint-Thurian de Quintin ${ }^{37}$. Seule la toile semble pouvoir expliquer une telle fortune et le Vieux-Bourg est, du reste, une des seules paroisses dont les comptes conservent une catégorie indépendante dans la charge pour le fil et la toile, rubrique dont la valeur est très fluctuante - entre 19 et 177 livres - mais qui est bel et bien une présomption de prospérité toilière. Or, Jean Martin ne

31. Jean Martin en dénombre huit dans les années 1780 (op. cit., p. 166).

32. Arch. dép. des Côtes-d'Armor, 20 G 57/58.

33. Dont le niveau ne dépasse pas 190 livres à la fin du XVIII ${ }^{e}$ siècle.

34. MARTIN, Jean, Toiles de Bretagne..., op. cit., p. 167.

35. Arch. dép. des Côtes-d'Armor, 20 G 679.

36. MARTIN, Jean, Toiles de Bretagne..., op. cit., p. 232.

37. MouchEt, Aurélie, Espace urbain et vie religieuse : la paroisse Saint-Thurian de Quintin aux $\mathrm{XVII}^{\mathrm{e}}$ et $\mathrm{XVII{ } ^ { \mathrm { e } }}$ siècles, mémoire de maîtrise, université de Rennes 2 , sous la direction de Georges Provost, 2003, 356 p. 
mentionne aucun marchand de toiles dans cette paroisse où, au vu de la richesse de la fabrique, on aurait pu s'attendre à les trouver en force étant donné que ce sont eux qui s'enrichissent le plus. Le cas du Vieux-Bourg offre donc le cas de figure original d'une richesse indéniable, probablement liée pour partie à la manufacture toilière mais en l'absence d'un milieu marchand important.

Graphique 3 - Valeur moyenne en livres des offrandes

faites à la fabrique du Vieux-Bourg

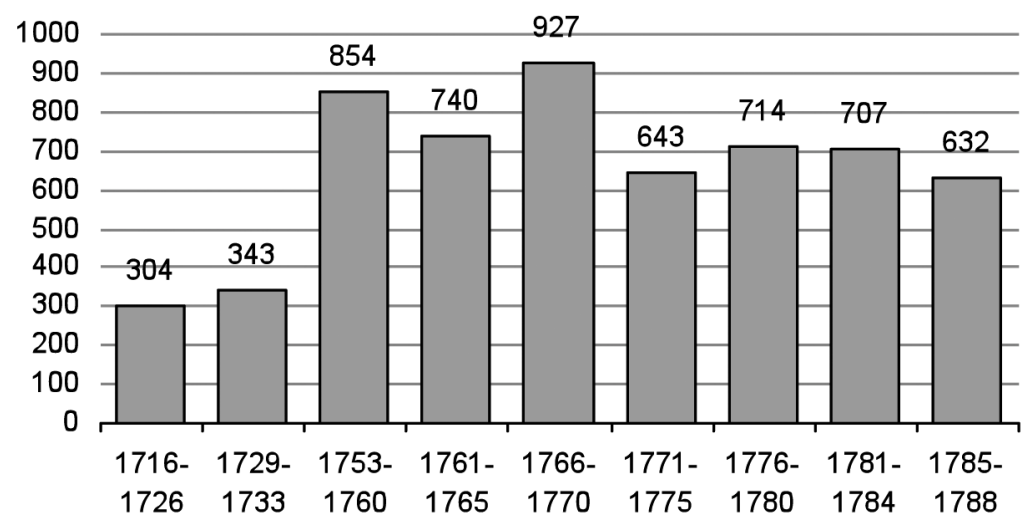

Les comptes de fabrique révèlent donc des situations complexes, et I'on ne peut se reposer sur eux seuls pour établir, en Centre-Bretagne, un lien entre les chantiers paroissiaux et la toile. Il faut faire appel à d'autres sources et recourir à une approche plus sociale en recherchant l'existence, dans les paroisses, d'un milieu de marchands de toiles.

\section{La domination des marchands de toile}

L'absence de marchands au Vieux-Bourg est d'autant plus étonnante que dans les paroisses qui profitent le plus de la prospérité toilière, la richesse de la fabrique coïncide avec un milieu marchand plus ou moins important mais réel. C'est le cas au Bodéo, au Quillio et à Saint-Thélo.

Pour retrouver les marchands, il faut souvent procéder à un croisement de plusieurs sources : les rôles de capitation, les livres de délibérations et les registres de marques apposées sur les balles de toile. Les rôles de capitation donnent une idée du niveau de fortune des notables de la paroisse, voire, dans le cas du Bodéo, de leur situation sociale. Les rôles les plus anciens, ceux de 1695 et $1697^{38}$, distinguent deux hommes

38. Arch. dép. des Côtes-d'Armor, 20 G 11. 
comme "marchands de toile blanche en gros » : Jean et Gilles Garnier, respectivement capités à 30 et 21 livres. En dessous figurent sept hommes désignés comme "marchands tisserands ", dont la capitation ne dépasse pas 10 livres 10 sols. Pour ce qui concerne Jean Garnier, on le retrouve encore par la suite, doté du titre de " sieur de Kérigant», sa capitation atteignant 40 livres en 1710, date du dernier rôle conservé. Son nom se retrouve dans les livres de délibérations: il siège au corps politique de la paroisse à 15 reprises entre 1711 et 1735 . II n'est pas le seul. La plupart des marchands de toiles du Bodéo, pourtant peu nombreux, se retrouvent dans le général, souvent pour plusieurs années : en 1715, 1717 et 1718, six délibérants sur douze sont marchands. Voilà qui en dit long sur leur dimension de notables locaux et leur domination de la vie paroissiale. La famille Garnier de Kérigant se retrouve également dans les registres de marques du marché de Quintin ${ }^{39}$. Ces registres signalent d'autres marchands du Bodéo comme les Morice.

Au Bodéo, les sources concordent ainsi remarquablement pour permettre d'évaluer le milieu marchand en nombre, en richesse et en influence. Saint-Thélo et le Quillio n'ont pas la même chance : les registres de marques des marchés d'Uzel et de Loudéac sont perdus et il est donc plus difficile d'établir la condition de marchand des notables de cette paroisse et de cette trève. Elle ne fait toutefois guère de doute dans la mesure où nous sommes ici au cœur même de la manufacture, là où l'activité était à son maximum et où le patrimoine monumental laissé par la toile est aussi le plus évident. Les comptes de fabrique de Saint-Thélo ont disparu mais ceux du Quillio montrent une croissance des offrandes dès la seconde moitié du XvII siècle. Au Quillio, la capitation révèle également un niveau de richesse élevé, indice qui doit être combiné avec la présence repérable des contribuables les plus fortement cotés dans les registres de délibérations du général de la paroisse. La capitation fournit un autre indice ponctuel puisque certains notables acquittent une petite somme supplémentaire pour leur «facteur ${ }^{40}$ ». Or, les facteurs servaient de commissionnaires aux grands marchands de toiles ${ }^{41}$. Cela semble confirmer la fonction de marchand des sieurs Kermarec-Ollitrault, Cosquer du Coédic, Brince-Guillo, Lohan-Guillo et Duparc-Ollitrault, tous du Quillio.

L'usage de titres, que l'on sait croissant à partir de la seconde moitié du $x v I^{\mathrm{e}}$ siècle $^{42}$, constitue un autre indice pertinent pour identifier des marchands de toiles : ainsi pour Garnier de Kérigant au Bodéo. Leur apparition est en effet caractéristique de l'ascension sociale des marchands de toiles: les marchands quintinais ont montré l'exemple dès les années 1650 . Les registres de délibérations du Quillio et de Saint-Thélo font mention, pour certains membres des deux généraux, de titres qui se transmettent à leurs

39. Arch. dép. des Côtes-d'Armor, B 3637.

40. Arch. dép. des Côtes-d'Armor, 20 G 705.

41. MARTIN, Jean, Toiles de Bretagne..., op. cit.

42. Ibidem, p. 235-236. 
descendants ${ }^{43}$. Les sources laissent ainsi apparaître 18 sieurs au Quillio et 27 à Saint-Thélo ${ }^{44}$. Au Quillio, il est possible de recouper en partie la liste des sieurs avec les rôles de capitation. On voit ainsi émerger un groupe d'hommes qui présentent toutes les caractéristiques des grands marchands et dominent la vie paroissiale. Ils acquittent une forte capitation, disposent pour certains d'entre eux d'un facteur, dominent le général où ils siègent parfois de façon quasi permanente; ce sont aussi des " sieurs" qui transmettent le titre à leurs fils de même que, bien souvent, leur place au général. On peut ainsi suivre de véritables dynasties. C'est la concordance de tous ces éléments qui laisse penser que l'on a affaire à de grands marchands de toiles.

L'un des exemples les plus remarquables est celui de la famille Cosquer du Coédic, dans la trève du Quillio. Les sources permettent d'identifier quatre de ses membres qui se succèdent tout au long du XvIII ${ }^{\mathrm{e}}$ siècle. On retrouve les trois premiers dans le corps politique de la trève ${ }^{45}$ : Ellouan siège entre 1712 et $1722^{46}$, puis son fils Olivier entre 1717 et 1756 et enfin Hervé, le petit-fils, entre 1750 et 1766 . Les rôles de capitation indiquent un quatrième Cosquer du Coédic, prénommé également Olivier, imposé à partir de $1774^{47}$. Sans doute siégeait-il aussi au général mais l'absence des délibérations postérieures à cette date empêche de l'affirmer. II s'agit ici de l'une des plus influentes familles de la trève au XVIII ${ }^{\mathrm{e}}$ siècle, avec une succession particulièrement bien servie par les sources. De plus, un acte consigné dans les délibérations de 1761 désigne clairement le sieur Hervé Cosquer du Coédic comme un marchand de toiles « bretagnes ${ }^{48}$ ».

Deux de ces grands notables bénéficient même de la mention « noble homme » lorsqu'ils sont désignés dans les délibérations : le sieur François Glais de la Villeaupré à Saint-Thélo et le sieur du Cosquer du Coédic au Quillio. D'autres encore maintiendront suffisamment leur influence pendant la Révolution puis au XIX $x^{\mathrm{e}}$ siècle, malgré le déclin de la manufacture, pour accéder à la fonction de maire : Boscher de Langle à Saint-Thélo ou encore Guillo-Lohan au Quillio.

Les exemples ci-dessus confirment qu'il est possible d'identifier un véritable milieu marchand dans certaines paroisses rurales de la manufacture des "bretagnes». Tous ne sont donc pas concentrés dans les trois villesmarchés de Quintin, Loudéac et Uzel. Ainsi, certaines paroisses rurales reçoivent tout de même une part de cette prospérité toilière qui permet à

43. Arch. dép. des Côtes-d'Armor, 20 G 602 et 20 G 702.

44. Dans les années 1780, Jean Martin a recensé 34 marchands au Quillio et 14 à SaintThélo (Martin, Jean, op. cit., p. 166).

45. Arch. dép. des Côtes-d'Armor, 20 G 702.

46. Les périodes indiquées ne sont que les bornes chronologiques de leur présence au général : entre ces deux dates extrêmes, ils ne siègent évidemment pas toutes les années.

47. Arch. dép. des Côtes-d'Armor, 20 G 705.

48. Arch. dép. des Côtes-d'Armor, 20 G 702, délibération du 18 octobre 1761. Le Quillio fournit deux autres exemples intéressants de "dynasties marchandes » : celui des familles Guillo-Lohan et Kermarec-Ollitrault. 
leurs fabriques de s'enrichir. L'impact de la toile n'est pas alors uniquement financier : la vie de la paroisse se trouve influencée par les décisions de ces grands marchands de toiles qui dominent le général, y compris ce qui concerne les chantiers paroissiaux.

\section{L'ambition fabricienne}

L'argent que les marchands tirent de leur commerce toilier, ils ne l'investissent pas seulement dans leurs belles et grandes demeures, mais ils savent aussi se montrer généreux envers leur paroisse et leur église. L'orgueil du marchand, fier de sa réussite sociale et désireux d'en faire état, se confond ici avec l'orgueil paroissial. La générosité du marchand sert I'un aussi bien que l'autre. S'y ajoute aussi, sans doute, une part de piété.

C'est à Saint-Thélo que se rencontrent les exemples les plus probants. Le calvaire financé en 1731 par Yves Glais, sieur de la Villeblanche, et qu'il fait placer juste devant la porte de l'église (photo 1) témoigne bien de cet orgueil individuel du marchand de toile affichant sa réussite, mais qui contribue aussi à embellir l'église et son enclos, donc profite à la paroisse toute entière. De la même façon, Mathurin Rolland, sieur de Châteaublanc, participe au financement en 1676 de la réalisation du retable de la confrérie des Agonisants : I'inscription sur le retable en atteste ${ }^{49}$. Le tableau du retable (photo 2 ) présente un notable en prière au pied du mourant, et ce notable est le seul personnage à être représenté vêtu à la mode de la fin du $x \mathrm{XII}^{\mathrm{e}}$ siècle, ce qui laisse penser qu'il s'agit de la représentation du marchand qui a financé le retable. Quant à François Glais, sieur de la Villeaupré, il avance, par " une pieuse bienveillance », plus de 6400 livres pour la construction de la chapelle des fonts baptismaux à partir de $1731^{50}$. C'est un prêt à long terme puisque le général n'achèvera de le rembourser qu'en 1746, et sans intérêt, ce qui, de la part d'un marchand de toiles d'envergure, n'est pas un geste anodin. Là aussi, l'orgueil personnel sous-tend l'action de Villeaupré : cette chapelle des fonts servira aussi de chapelle mortuaire à sa famille.

Sans être toujours aussi spectaculaire, la participation des marchands aux chantiers paroissiaux peut prendre d'autres formes. À un premier niveau, elle tient déjà à leur présence dominante au sein du général - que ce soit à Saint-Thélo, au Quillio ou encore au Bodéo - qui coïncide avec une indéniable ambition fabricienne : en l'occurrence, d'importants programmes de chantiers paroissiaux dont il semble bien qu'ils ont été prévus plusieurs années à l'avance dans un projet d'ensemble, sans que cela ne soit clairement exprimé.

Ainsi, au Bodéo, un important programme mené dans le premier quart du $X V I I I^{e}$ siècle conduit à un remodelage complet de l'église, reconstruite

49. «Donné par H : gens Pierre Moulnier sieur de Guerochoux \& Mathurin Rolland sieur de Château : Blanc : 1676 : et :F : P:G : Le : Poder. »

50. Arch. dép. des Côtes-d'Armor, 20 G 702. 
en $1705^{51}$ et complétée par un porche en 1708. Pour le décor intérieur, le général finance d'abord la construction du retable du maître-autel en 1717 pour 600 livres puis sa peinture et sa dorure en 1724. Avec l'aide des fonds de la confrérie du Rosaire, il achève la peinture des lambris pour 1500 livres entre 1724 et $1726^{52}$. Ce programme financièrement lourd est réalisé en quelques années seulement, par une petite paroisse qui, malgré une conjoncture alors favorable, ne dispose pas de fonds illimités. Le cas est révélateur de la façon dont de tels chantiers sont financés : par un processus cyclique d'accumulation et d'investissement. Les caisses de la fabrique sont vides en 1706, conséquence probable de la reconstruction de l'église; dans les années suivantes, le général attend que la réserve se reconstitue, environ à hauteur de mille livres, puis finance un grand chantier qui vient réduire cette somme de moitié (1717 et 1724). Seule cette accumulation, favorisée par un contexte fabricien et toilier favorable, permet de financer de tels programmes. Un tel cycle semble bien indiquer que le général avait un projet d'ensemble quant aux chantiers de son église.

Le phénomène est encore plus remarquable au Quillio (photo 3) dans la seconde moitié du XVII ${ }^{\mathrm{e}}$ siècle. Les années $1655-1671^{53}$ sont particulièrement actives et donnent là aussi l'impression d'un plan d'embellissement prévu à l'avance et financé au fur et à mesure par une accumulation, efficacement alimentée par l'augmentation des offrandes. Ainsi, quatre retables, une chaire, un tabernacle, des tableaux ou encore la peinture du jubé sontils réalisés. Chacune de ces dépenses ne dépasse pas quelques centaines de livres mais elles reviennent avec fréquence; s'y ajoutent par surcroît 800 livres en ornements divers, ainsi que 1000 livres pour la construction du dôme de l'horloge entre 1667 et 1668. Une telle liste met donc en évidence un chantier paroissial presque permanent pendant cette quinzaine d'années. La fabrique gagne alors plusieurs centaines de livres par an, profitant de l'afflux de dons consécutif à la création d'une quête spéciale dite de « Notre Dame » qui double les offrandes habituelles à partir de 1668. Malgré cela, sous l'effet de tous ces chantiers, le reliquat stagne. II ne commence à s'élever qu'après 1675 quand la frénésie de chantiers s'apaise : de 166 livres, il passe à 887 en 1681. Ici, le général n'attend même pas que l'accumulation fasse son œuvre, il investit quasiment au fur et à mesure que les dons arrivent.

D'autres éléments attestent encore de l'influence des marchands sur les chantiers paroissiaux. Quand il passe une commande, le général nomme des députés pour suivre le chantier de près, surveiller les artisans, les payer, assurer leur approvisionnement : ceux-ci s'avèrent souvent être des marchands. Les registres de délibérations du Bodéo offrent à nouveau un exemple des plus intéressants lors d'une âpre négociation avec le peintre

51. Les comptes de ces années étant perdus, on ignore combien cette reconstruction a pu coûter.

52. Arch. dép. des Côtes-d'Armor, 20 G 10.

53. Arch. dép. des Côtes-d'Armor, 20 G 703/704. 
pontivien Dupont au sujet de la peinture des lambris de l'église en 1724 . La négociation piétine faute d'un accord sur le prix et le général se décide finalement à envoyer un dernier négociateur, Jean Collin, sieur de Petit Bois, un marchand de toiles qui parviendra à obtenir l'accord du peintre pour 800 livres $^{54}$. De plus, le général fait appel à un architecte malouin, Jean Soulié ${ }^{55}$, pour assurer la reconstruction de l'église. Or, qui mieux que les marchands de toiles de la paroisse était susceptible de connaître un architecte malouin? Les marchands avaient en effet l'habitude de fréquenter Saint-Malo, port principal pour l'exportation des « bretagnes ${ }^{56}$ ».

Au Bodéo, à Saint-Thélo, au Quillio, il apparaît donc que les marchands de toiles sont, très impliqués dans les chantiers paroissiaux et s'activent à l'embellissement de leurs églises. Prenons garde toutefois à ne pas généraliser à l'ensemble de la manufacture des constats qui ne valent que pour ces trois seules paroisses : les lacunes des sources, la présence de certains contre-exemples invitent en effet à ne pas systématiser le lien entre prospérité toilière et chantiers paroissiaux.

\section{Un lien systématique?}

Malgré l'évidence des constats qu'elles autorisent, les deux paroisses et la trève évoquées plus haut ne relèvent-elles pas de l'exception plus que de la règle? L'historien y bénéficie d'une conjonction des sources et peut établir que tous les facteurs s'y rencontrent pour favoriser des chantiers paroissiaux d'envergure. Mais on ne saurait généraliser à partir de ces trois cas, tant il est clair que toutes les paroisses de la manufacture n'avaient ni une telle ambition, ni les moyens de la réaliser; et même si l'on se limite à celles qui soutiennent des programmes artistiques importants, il s'avère qu'elles n'ont pas toujours de marchands aux commandes du corps politique.

Une seule paroisse, le Vieux-Bourg, déjà mentionnée pour sa puissance fabricienne atypique, suffit à alerter de la complexité des choses. Ici, la seconde moitié du XVIII siècle est marquée par de très importants chantiers somptuaires ${ }^{57}$ : en 1754, le retable du maître-autel est réalisé (2 445 livres), l'année suivante, c'est la chapelle des fonts et son décor (1 200 livres), les dorures de l'église en 1764 (2515 livres), la réalisation de deux cloches entre 1780 et 1787 (4340 livres), sans compter de coûteux ornements : un soleil en 1768 (1 246 livres), une croix d'argent en 1774 (906 livres), ou encore une lampe d'argent en 1784 (1 453 livres). On touche là un niveau d'investissement inédit qui pourrait presque rivaliser avec ce que l'on rencontre dans les paroisses léonardes. Là encore, la concentration de fortes dépenses dans la décennie 1754-1764 laisse penser à un programme établi

54. Arch. dép. des Côtes-d'Armor, 20 G 10.

55. BouRde de LA Rogerie, Henri, Fichier Bourde de la Rogerie : artistes, artisans et ingénieurs en Bretagne, Bruz, Association pour l'inventaire Bretagne, 1998, notice 08185.

56. LesPaGnol, André, Messieurs de Saint-Malo..., op. cit., p. 426-442.

57. Arch. dép. des Côtes-d'Armor, 20 G 679. 
à l'avance par le général pour renouveler l'essentiel de la décoration intérieure de l'église. C'est aussi la période où le niveau des offrandes est le plus élevé ${ }^{58}$. Grâce à cela, et malgré les lourdes dépenses, la réserve financière se reforme très vite : ainsi, entre 1756 et 1758 , elle passe de 154 livres à 1035 livres. Le général profite évidemment de cette excellente conjoncture fabricienne pour lancer presque de front toutes ces dépenses somptuaires. On retrouve donc toujours les mêmes éléments. Le seul qui manque ici est la présence d'un milieu marchand.

Un autre exemple, celui de la trève de La Harmoye, montre que, même sans milieu marchand pour diriger et dynamiser les affaires de la paroisse, les généraux sont capables d'entreprendre, d'investir et de planifier des chantiers paroissiaux. Par les registres de capitation de sa paroisse-mère, Le Bodéo, on sait qu'il n'y avait aucun marchand de toiles à La Harmoye ${ }^{59}$. Peut-être y en eut-il mais pas de façon durable : une délibération du général de la trève évoque, en effet, en 1752 " plusieurs bonnes maisons marchandes devenues insolvables ${ }^{60} »$. Pourtant, entre 1760 et 1775 , le général ne cesse d'investir pour réaliser ce qui semble être un programme cohérent : en 1760, il fait construire une chapelle du côté de l'Évangile, pour laquelle il fait ensuite réaliser et peindre un retable entre 1763 et 1766 . Puis, après avoir fait réaliser une chaire en 1767 , il poursuit le chantier en faisant reconstruire la chapelle de l'Épître en 1770 et réaliser son retable en 1772. Enfin, il achève la décoration du chœur en faisant dorer et peindre le retable du maître-autel et le tabernacle en 1775. Il y a là une réelle ambition fabricienne, et qui ne paraît rien devoir aux marchands de toiles, en tout cas de façon directe.

Pour dépasser le cadre d'exemples forts mais ponctuels, et dont on peut se demander s'ils sont vraiment représentatifs, l'analyse globale, à l'échelle de toute la manufacture trouve à nouveau sa pertinence. II faut tout d'abord rappeler qu'un grand nombre de réalisations en matière d'édifices religieux, à l'échelle de la manufacture, datent bien de l'époque de la prospérité toilière ${ }^{61}$, ce qui induit que même si l'enrichissement est très inégal, il permet globalement un renouvellement du patrimoine religieux des paroisses de la manufacture.

Malgré les lacunes des sources, 116 chantiers monumentaux peuvent être recensés dans les différentes paroisses et trèves de la manufacture entre 1650 et 1825 . Bien sûr, il importe de distinguer les grands chantiers de reconstruction, qui représentent un investissement lourd, de ceux, moins onéreux, qui se limitent à des réaménagements, embellissements ou agrandissements comme l'ajout d'une chapelle latérale ou d'une sacristie. Or, si I'on s'intéresse plus particulièrement aux reconstructions, on remarque

58. Ce qui peut être lié aussi à ce contexte de grandes dépenses : on demande alors aux paroissiens de faire un effort et de se montrer plus généreux qu'à l'accoutumée.

59. Arch. dép. des Côtes-d'Armor, 20 G 11.

60. Arch. dép. des Côtes-d'Armor, 20 G 107.

61. MARTIN, Jean, Toiles de Bretagne..., op. cit., tableau p. 253. 
Graphique 4 - Les chantiers de reconstructions d'églises (nombre de chantiers)

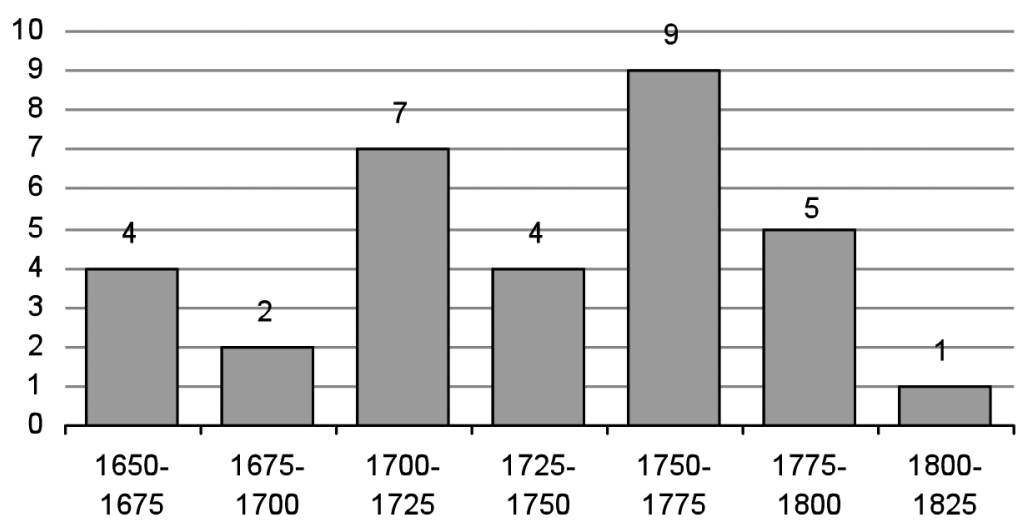

Graphique 5 - Les chantiers de constructions de clochers (nombre de chantiers)



qu'il y en eut 32 dans la manufacture entre 1650 et 1825, c'est-à-dire qu'à la grande époque de l'activité toilière, plus de la moitié des paroisses de la manufacture ont fait rebâtir leur église. La fréquence des constructions de clochers confirme ce mouvement, de façon d'autant plus significative que le clocher revêt une dimension particulière : il est l'édifice annexe le plus important de l'église et aussi le symbole de l'orgueil paroissial. La construction d'un clocher coûte cher et fait souvent l'objet d'un chantier spécifique. On en recense ainsi 32 dans les paroisses de la manufacture et encore est-ce sans compter ceux qui furent réalisés dans le cadre d'une reconstruction totale. 
On ne peut donc douter du fait que la période est particulièrement favorable à des chantiers paroissiaux de grande envergure. Si l'on observe de plus près la répartition chronologique de ces grands chantiers, le lien avec la prospérité toilière semble même se confirmer davantage. En effet, l'essentiel des chantiers de reconstruction (20 sur 32) sont réalisés entre 1700 et 1775 , c'est-à-dire en pleine période d'apogée de la manufacture. La même observation se dégage de la chronologie des clochers. A contrario, le déclin observé par la suite trouve également sa justification dans la conjoncture toilière, désormais dégradée en raison des graves difficultés consécutives, notamment, à l'augmentation des droits de douanes sur les toiles en Espagne ${ }^{62}$. Avec moins de revenus, les paroisses n'ont plus les moyens de financer des chantiers aussi lourds, sauf à disposer de fonds résultant de l'accumulation de la période précédente, d'où la réalisation tardive de quelques chantiers d'envergure. Un « effet-retard » reste donc possible une fois la grande prospérité passée. Un même décalage pouvait d'ailleurs s'observer en amont, dans la relative rareté des chantiers du XVII ${ }^{e}$ siècle (six seulement) : malgré l'enrichissement lié à la toile, il fallait en effet du temps aux paroisses pour accumuler l'argent nécessaire à un chantier d'envergure, et cela justifierait que le démarrage réel des chantiers paroissiaux ne se fasse qu'au XVIII siècle.

La cartographie des chantiers paroissiaux, classés selon leur date de réalisation, illustre finalement bien, dans sa réalité et ses nuances, le lien entre ambitions fabriciennes et prospérité toilière (voir cartes en annexe). Le mouvement de la prospérité se retrouve certes dans la densité particulière des chantiers de la seconde moitié du xvIII siècle, en particulier dans le pays de Loudéac qui devient alors le centre de gravité de la manufacture. Ce même mouvement peut expliquer aussi, à l'inverse, le manque de grandes réalisations dans la partie sud-est de la manufacture : I'activité toilière ne s'y est développée que tardivement dans le xvIII ${ }^{e}$ siècle, trop tard pour que l'enrichissement finance de grands chantiers. Encore ne peut-on écarter tout à fait I'hypothèse que cette absence de chantiers reflète aussi les lacunes des sources, particulièrement avérées pour les paroisses de ce secteur.

Si les paroisses situées autour de l'axe structurant des trois marchés de Quintin, Uzel et Loudéac lancent beaucoup de chantiers, les autres ne sont pas complètement en reste. La répartition des chantiers est même plutôt homogène si on la rapporte à l'inégalité, signalée plus haut, des richesses fabriciennes. Si certaines paroisses frontalières de la manufacture n'ont sans doute connu qu'une activité toilière trop tardive ou trop marginale pour en ressentir vraiment les effets bénéfiques, cela ne semble pas les avoir empêché de financer des chantiers, même modestes. La foi et la nécessité suffisent à lancer ces chantiers. Dans les paroisses suffisamment enrichies par la prospérité toilière, ceux-ci sont simplement plus ambitieux. II ne faut donc pas conclure trop vite à un impact mécanique de

62. MARTIN, Jean, Toiles de Bretagne..., op. cit., p. 200. 
la prospérité toilière sur les chantiers paroissiaux. Celui-ci est réel mais il ne peut rendre compte de toutes les situations qui se rencontrent dans les paroisses de la manufacture.

La prudence est d'autant plus de mise qu'une dernière nuance s'impose dans le cas de paroisses dont la fortune relative doit moins à la toile qu'à un pardon ou un pèlerinage particulièrement réputé, rapportant des revenus non négligeables mais provenant en partie de l'extérieur de la paroisse. Tel est le cas de La Prenessaye, où se développe l'important sanctuaire de Notre-Dame-de-Toutes-Aides à Querrien dès la seconde moitié du XvII ${ }^{\mathrm{e}}$ siècle alors que la paroisse est très en marge du commerce toilier ${ }^{63}$. Grâce à ce seul pèlerinage, ce sont des centaines de livres qui viennent gonfler chaque année les comptes de la chapelle ${ }^{64}$. Un même facteur religieux explique également l'effondrement que subit la courbe des offrandes faites à l'église de Boqueho après 1721 : alors que la moyenne était à 108 livres entre 1712 et 1723 , elle ne dépasse plus la trentaine de livres après cette date. La raison en est que les comptes ne mêlent plus, après 1721 , les reliquats de l'église avec ceux de la chapelle Notre-Dame de Pitié qui tient désormais un compte indépendant. La fortune de l'église décline alors fortement et repose essentiellement sur les rentes. Quant à la chapelle, elle continue pour sa part à recevoir de nombreux dons avec des moyennes qui varient entre 102 et 198 livres $^{65}$ et le détail de la " charge » montre bien que c'est le jour du pardon qu'affluaient en grande partie ces offrandes ${ }^{66}$. Enfin, c'est la chapelle qui assure les plus grandes dépenses somptuaires et aide financièrement l'église à l'occasion ${ }^{67}$.

Les paroisses de la manufacture des toiles « bretagnes » ont donc connu, au temps de la prospérité, des chantiers importants. À première vue, le même phénomène que dans les paroisses du Léon toilier se retrouve un siècle plus tard, quoique de façon moins spectaculaire. II n'en reste pas moins qu'ici aussi certaines paroisses connaissent une réelle augmentation des offrandes et profitent de cet enrichissement pour financer de véritables programmes d'investissement. L'ambition fabricienne se prolonge même au-delà de la prospérité, après le déclin de la manufacture : I'ancienne trève

63. Arch. dép. des Côtes-d'Armor, 20 G 828.

64. Il en est ainsi pour les premières années suivant la création de la chapelle. Hélas, les seuls comptes conservés concernent la période 1653-1656. II est impossible de dire comment évolua ensuite la conjoncture fabricienne de cette chapelle.

65. Arch. dép. des Côtes-d'Armor, 20 G 13.

66. C'est aussi le cas pour l'église tréviale du Leslay : c'est la ferveur autour des pardons de Saint-Symphorien et de la Madeleine qui, plus que la toile, explique la bonne fortune de la trève.

67. Ainsi le trésorier de la chapelle verse 731 livres à la fabrique de l'église en 1749 afin qu'elle puisse acheter une croix et un encensoir. On retrouve le même phénomène entre la chapelle Sainte-Suzanne et l'église paroissiale de Mûr (voir GWLADYs, Thomas, La vie paroissiale à Mûr et à Saint-Guen au XVIII ${ }^{\mathrm{e}}$ siècle (vers 1680-1790), mémoire de maîtrise, université de Rennes 2, sous la direction de Georges Provost, 2000, 168 p.). 
du Quillio en donne un bon exemple puisqu'elle continue à enrichir son église au XIXe siècle en acquérant une partie du mobilier de l'abbaye de Bon Repos (cliché 4), dans un contexte économique pourtant difficile.

Les chantiers paroissiaux doivent beaucoup à la fortune de la fabrique mais aussi aux initiatives du général. Quand ce sont les marchands de toiles qui le dominent, on peut prendre la mesure de l'influence financière et décisionnelle de la toile sur les chantiers paroissiaux : fortune, audace et orgueil marchand se conjuguent alors. Il en va ainsi au Quillio, au Bodéo et à Saint-Thélo, où le lien entre prospérité toilière et chantiers paroissiaux apparaît aussi évident que dans les paroisses léonardes de l'âge d'or.

Il faut toutefois se garder de systématiser le parallélisme : la toile n'explique pas tout ici. Les courbes d'offrandes illustrent une prospérité très inégale. Les marchands de toiles, qui concentrent les richesses et ont un rôle décisionnel fort, sont eux-mêmes très inégalement répartis. Et pourtant la moitié des paroisses de la manufacture ont bien reconstruit leur église à l'époque de la prospérité et la répartition générale des chantiers est bien plus homogène que celle des profits de la toile. C'est que dans cette manufacture, l'argent qui finance les chantiers ne vient pas toujours de la toile ni des offrandes. Quelques paroisses bénéficient de pardons ou de pèlerinages qui gonflent leur fortune fabricienne, d'autres ont simplement beaucoup de rentes et peu de dons, comme certaines paroisses de la zone des «noyalles ${ }^{68}$ ». Autant d'éléments qui tendent à prouver que le lien entre prospérité toilière et chantiers paroissiaux, via les offrandes, ne doit pas être posé en postulat mais examiné au cas par cas. Et il en va de même pour le rôle des marchands : les importants programmes d'investissements du Vieux-Bourg et de La Harmoye n'ont pas été décidés par des marchands de toiles.

Ainsi, au sein même de la manufacture des "bretagnes ", l'impact de la toile sur les chantiers paroissiaux s'avère-t-il complexe à saisir. À ce titre, il conviendrait sans doute d'élargir les recherches aux paroisses extérieures à la manufacture à des fins de comparaison pour approfondir ces conclusions et éclairer davantage le lien entre toile et chantiers paroissiaux.

68. Lagadec, Yann et PoInteAu, Delphine, « La proto-industrie... », op. cit. 
Carte 1 - Localisation des principales paroisses et des villes-marchés de la manufacture des toiles « bretagnes » (les noms des villes-marchés sont indiquées en majuscules)

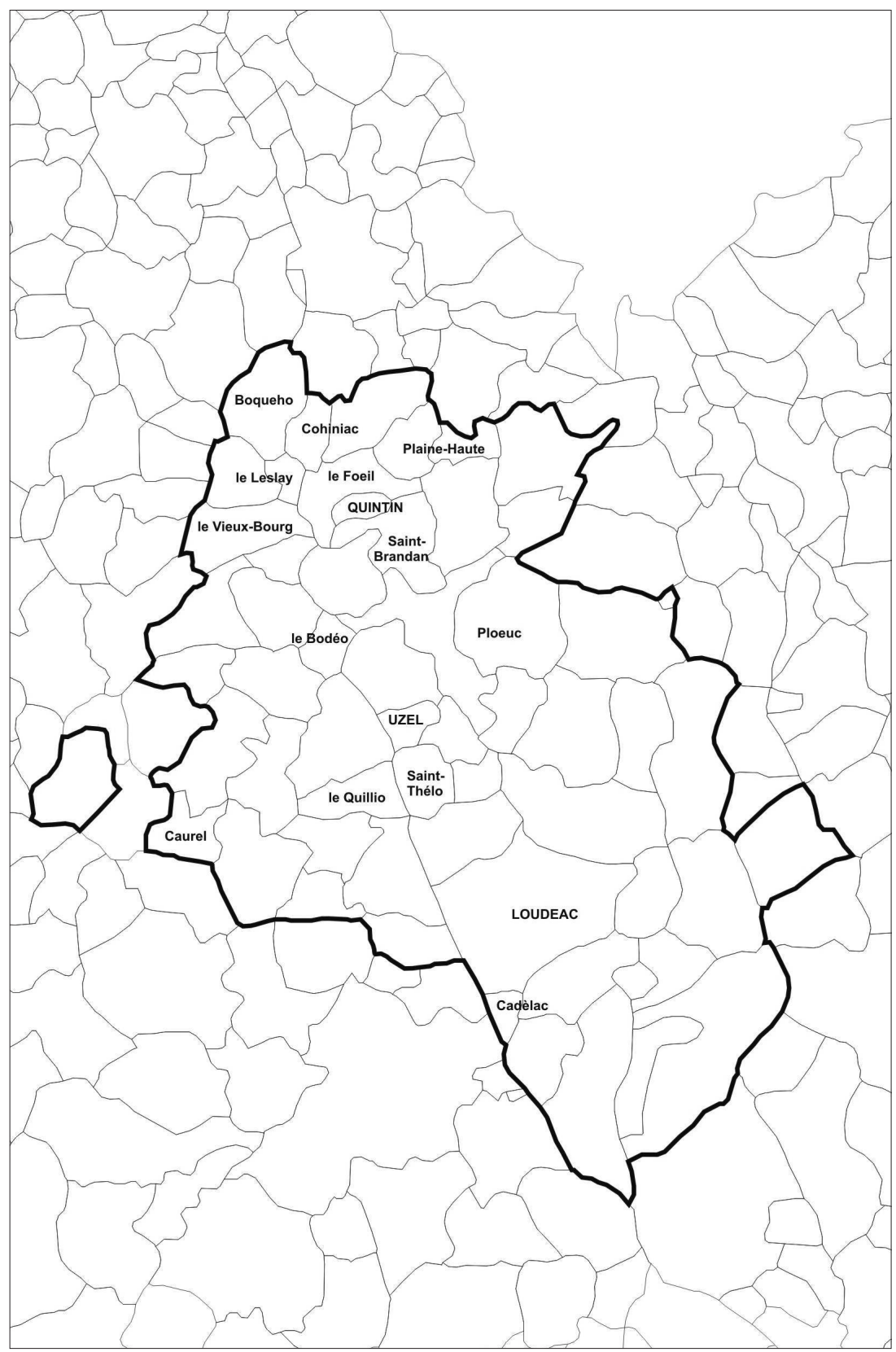


Carte 2 - Les reconstructions d'églises dans la région de la manufacture des toiles « bretagnes » entre 1650 et 1800

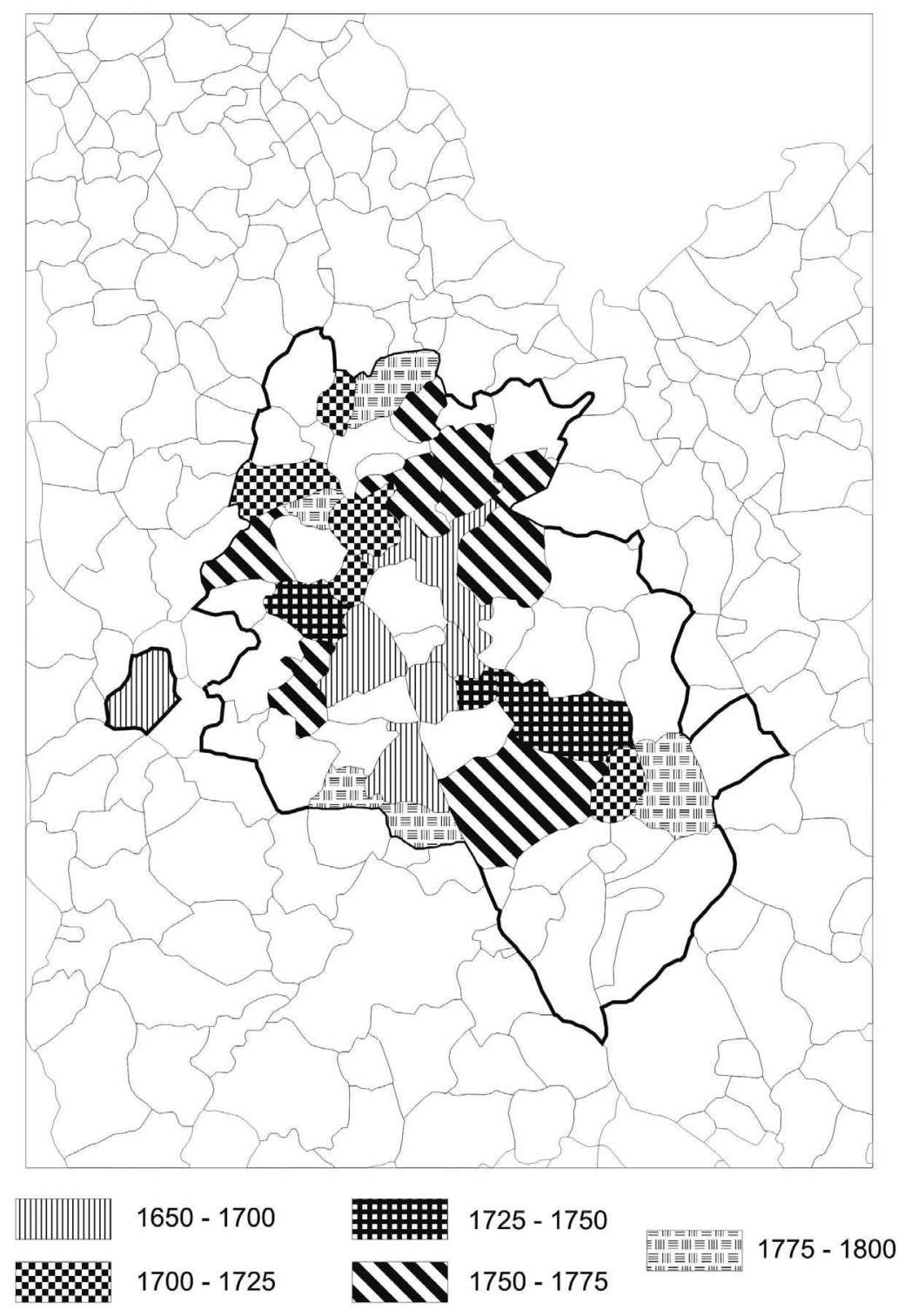

Limite de la manufacture

$$
0
$$

20 $30 \mathrm{Km}$ 
Carte 3 - Les constructions de clochers dans la région

de la manufacture des toiles « bretagnes » entre 1650 et 1800

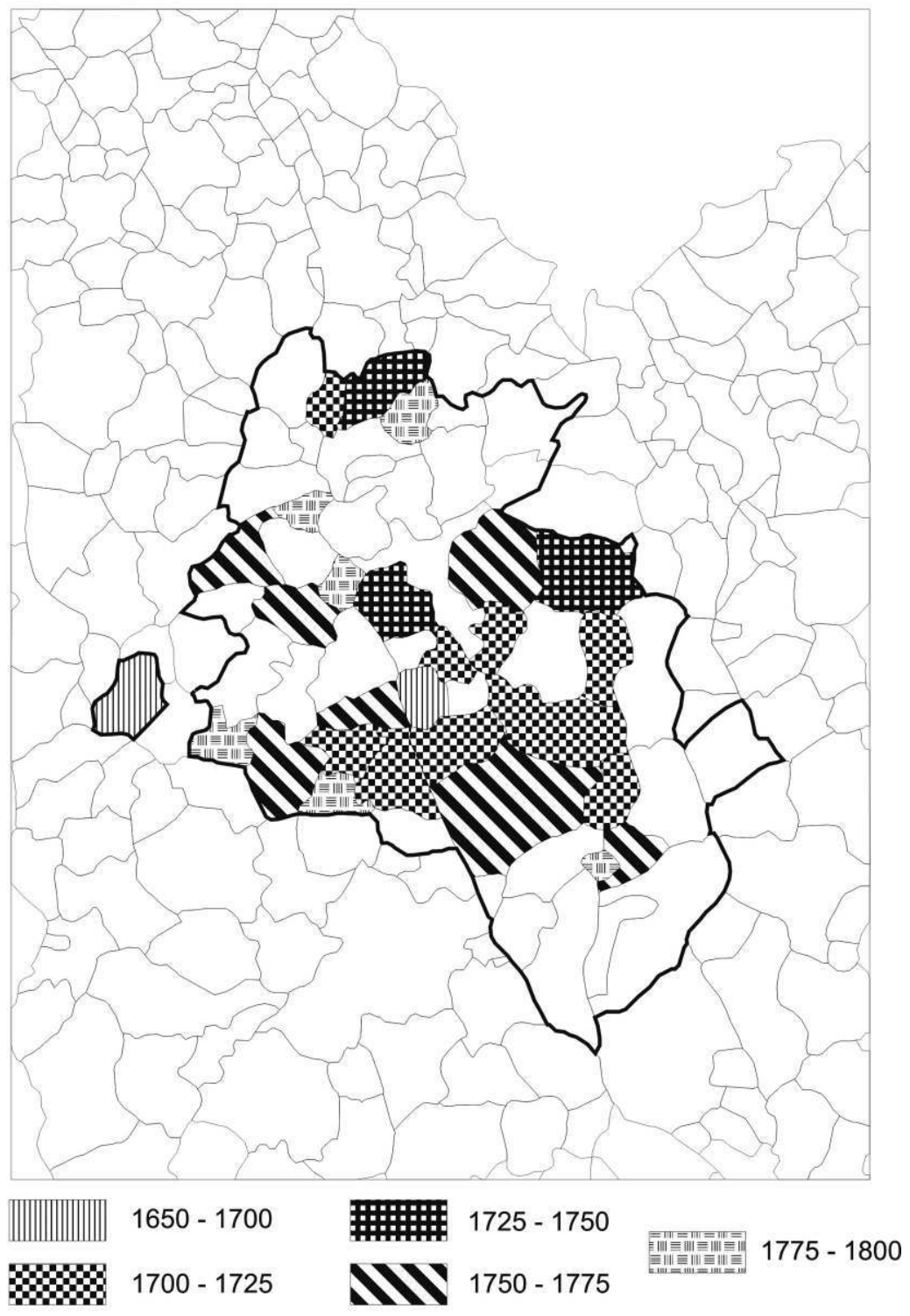

Limite de la manufacture

0 
Photo 1 - Saint-Thélo, église : calvaire érigé par Yves Glais en 1731 (cliché A. Guillemot, avril 2007)



Photo 2 - Saint-Thélo, église : tableau de la Bonne Mort (cliché Inventaire Bretagne)

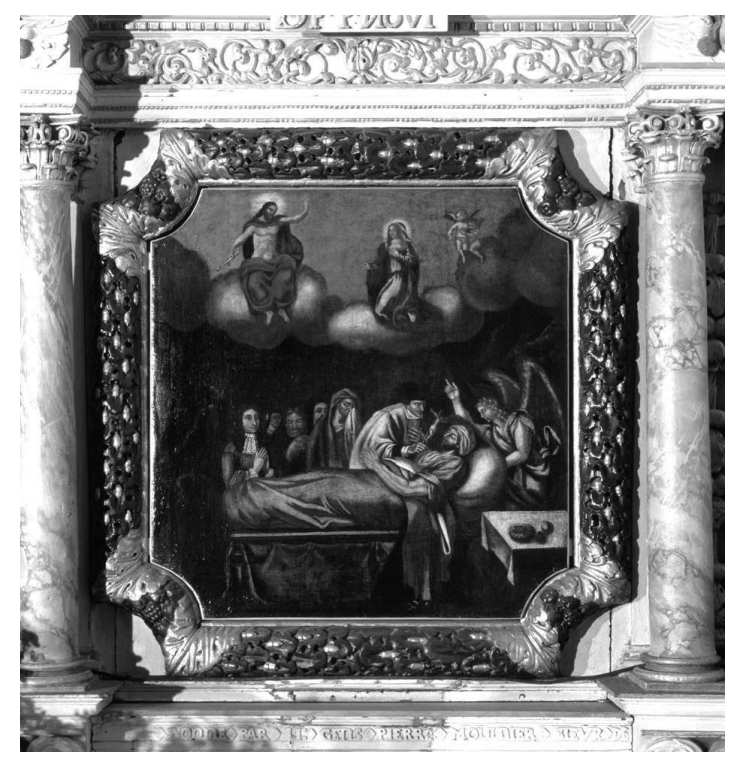


Photo 3 - Le Quillio, église et portail de l'enclos

(cliché A. Guillemot, avril 2007)
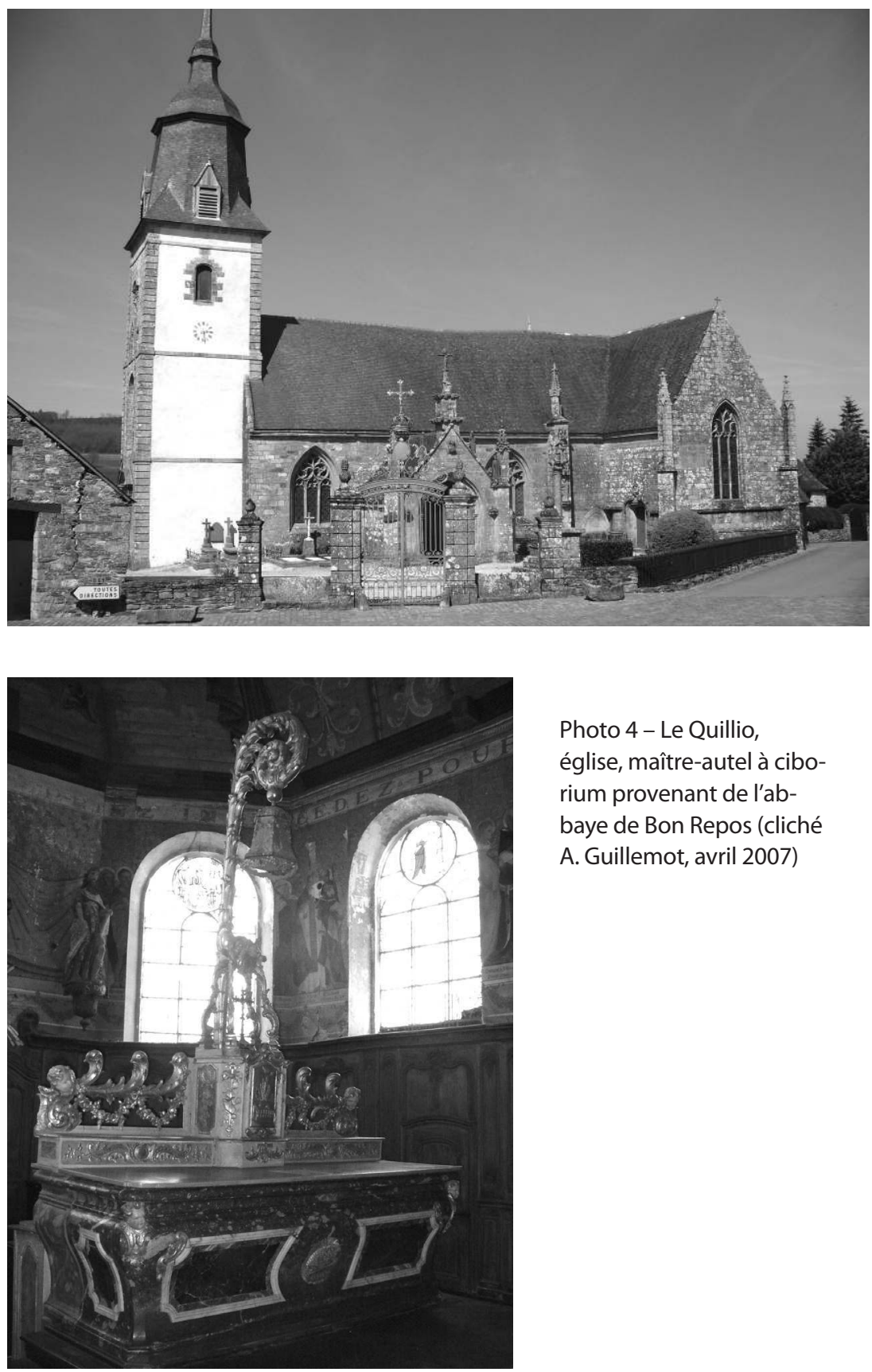

Photo 4 - Le Quillio, église, maître-autel à ciborium provenant de l'abbaye de Bon Repos (cliché A. Guillemot, avril 2007) 
RÉSUMÉ

Le dynamisme toilier que connaît la manufacture des toiles " bretagnes »

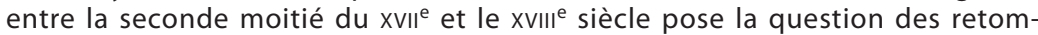
bées d'un tel commerce sur le patrimoine religieux. Un grand renouvellement des édifices religieux à l'époque faste de la manufacture ainsi que l'augmentation des offrandes dans certaines paroisses combinée à la présence d'un milieu marchand influent et dynamique semblent confirmer l'existence d'un lien entre prospérité toilière et chantiers paroissiaux.

Ce lien, réel, doit cependant être nuancé. Les marchands, principaux bénéficiaires du commerce toilier, ne sont pas présents partout, de même qu'il existe de grandes inégalités dans l'enrichissement des différentes paroisses. Or, cela n'empêche pas une certaine homogénéité dans la répartition des chantiers à l'échelle de la manufacture. L'impact du commerce toilier sur ces chantiers paroissiaux reste donc complexe à saisir.

\section{ABSTRACT}

The linen cloth industry which made bretagnes enjoyed a great dynamism from the middle of the 17th century to the end of the 18th century, an economical fact which leads us to wonder about a link between the dynamism of the cloth factories and church patrimony. Indeed, the period concerned by this economic development coincided with the building of many churches and a remarkable increase of the offerings in certain parishes. This observation tends to prove the existence of a relation between an influential merchant group and the golden age of church building.

Despite the evidence of a strong link, we must tone down this statement: merchants are not present everywhere, and there is a great diversity in the wealth of parishes; this underlines the complexity of the question, for there is indeed a certain homogeneity in the distribution of the building yards, as connected with the cloth industry. The impact of this particular trade on the "golden age of church building" remains hard to understand completely. 
